\title{
Etiologia, epidemiologia e manifestações clínicas da paracoccidioidomicose
}

\author{
Etiology, epidemiology e clinical manifestations \\ of paracoccidioidomycosis \\ Etiología, epidemiología y manifestaciones clínicas de paracoccidioidomicosis
}

\author{
Elerson GAETTI-JARDIM JÚNIOR ${ }^{1}$ \\ Lira Marcela MONTI ${ }^{2}$ \\ Ellen Cristina GAETTI-JARDIM ${ }^{3}$ \\ ${ }^{I}$ Departamento de Patologia e Propedêutica Clínica, Faculdade de Odontologia de Araçatuba, UNESP \\ Univ. Estadual Paulista, Araçatuba-SP, Brasil \\ ${ }^{2}$ Disciplina de Cirurgia Bucomaxilofacial Faculdade de Odontologia, UFMS \\ Univ. Federal do Mato Grosso do Sul \\ Especialista,Mestre, Doutora e Pós-Doutora em Cirurgia e Traumatologia Bucomaxilofacial. \\ Especilista e Mestre em Estomatologia
}

\begin{abstract}
Resumo
As últimas décadas presenciaram uma modificação significativa na epidemiologia da paracoccidioidomicose. O presente estudo discute retrospectivamente o perfil dos pacientes com essa enfermidade, destacando o papel do cirurgião-dentista no seu diagnóstico, bem como as modificações na distribuição dos casos dessa doença. Observou-se um aumento na notificação de casos dessa micose profunda nas regiões Norte e Centro-Oeste do Brasil, em particular nas frentes agrícolas. Estudos mostraram que outras espécies animais podem atuar como reservatórios dos patógenos do gênero Paracoccidioides, desenvolvendo, em alguns casos, a própria enfermidade. Estudos genéticos também evidenciaram que $P$. brasiliensis mostrava heterogeneidade significativa e alguns genogrupos passaram a compor nova espécie, $P$. lutzii, mais frequente na região amazônica. Os autores também discutem o papel da ocupação profissional e fatores hormonais no desenvolvimento de infecções e da enfermidade clinicamente caracterizada devido a Paracoccidioides sp. Além das transformações no conhecimento da doença propriamente dita, o estudo reforçou a importância do cirurgião-dentista no diagnóstico dessa enfermidade.
\end{abstract}

Descritores: Paracoccidioidomicose; Mucosa bucal; Manifestações Bucais; Epidemiologia.

\begin{abstract}
The last decades have seen profound modifications in the epidemiology of paracoccidioidomycosis. This study aimed to retrospectively discuss the profile of patients presenting this disease, highlighting the role of the dentist in the diagnosis as well the changes in the distribution of cases of this disease. There was an increase in notification of such mycosis in the North and Midwest regions in Brazil, particularly in the agricultural frontiers. Studies have shown that other animal species can act as a pathogen reservoir of genus Paracoccidioides and develop the clinical disease in some circumstances. Genetic studies also showed that $P$. brasiliensis evidenced significant heterogeneity and some genogroups were reunited into a new species, $P$. lutzii, more frequent in the Amazon region. The authors also discuss the role of occupation and hormonal factors in the development of these infections and clinically characterized disease due to Paracoccidioides sp. In addition to the changes in the knowledge about this condition, the study reinforced the importance of the dentist in the diagnosis of this disease.

Descriptors: Paracoccidioidomycosis; Mouth Mucosa; Oral Manifestations; Epidemiology.
\end{abstract}

\section{Resumen}

Las últimas décadas han visto un cambio importante en la epidemiología de la paracoccidioidomicosis y este estudio tuvo como objetivo discutir retrospectivamente el perfil de los pacientes con esta dolencia, destacando el papel del odontólogo en el diagnóstico y así como los cambios en la distribución de casos de esta enfermedad. Se observó un aumento en los casos de notificación de esta micosis en las regiones Norte y Centro-Oeste de Brasil, sobre todo en las frentes agrícolas. Los estudios han demostrado que otras especies animales pueden actuar como reservorios de patógenos del género Paracoccidioides, que pueden, en algunas circunstancias, el desarrollo de la enfermedad en sí. Los estudios genéticos también mostraron que $P$. brasiliensis tenía una heterogeneidad significativa y algunos genogrupos comenzó a componer nueva especie, $P$. lutzii, más frecuente en la región amazónica. Los autores también discuten el papel de la ocupación y los factores hormonales en la aparición de infecciones y enfermedades clínicamente caracterizado debido a Paracoccidioides sp. Además de los cambios en el conocimiento de la propia enfermedad, el estudio refuerza la importancia del dentista en el diagnóstico y de esta enfermedad.

Descriptores: Paracoccidioidomicosis; Mucosa Bucal; Manifestaciones Bucales; Epidemiología. 


\section{INTRODUÇÃO}

A paracoccidioidomicose é uma micose com manifestações mucocutâneas pronunciadas e, por vezes, sistêmicas ${ }^{1,2}$. Sua etiologia era creditada ao fungo dimórfico Paracoccidioides brasiliensis, mas em anos recentes verificou-se que sob essa denominação duas espécies distintas se encontravam reunidas e $P$. lutzii passou a ser reconhecido como espécie distinta, apresentando características próprias, como distribuição geográfica, virulência e diagnóstico laboratorial $^{3,4}$.

Esses fungos vivem como saprófitas no solo, onde formam micélios, integrados a comunidades microbianas complexas ${ }^{5}$, mas após a inalação de seus conídios, convertem-se em leveduras, a $37^{\circ} \mathrm{C}$, e podem originar essa micose sistêmica, produzindo lesões granulomatosas cuja evolução tem relação direta com a imunidade celular do paciente, com o inóculo e a distribuição geográfica dos acometidos ${ }^{1,2}$.

A paracoccidioidomicose apresenta caráter endêmico na América Latina, principalmente no Brasil, Argentina, Colômbia e Venezuela. Em nosso país, ela é mais frequente nas regiões Sul e Sudeste ${ }^{6-8}$, embora a expansão agrícola, observada a partir de 1980, esteja modificando esse cenário, acrescentando muitos novos casos na região Sul e Leste da Amazônia e nas áreas do bioma "savana",2-10.

Em âmbito nacional, a incidência anual dessa doença em áreas endêmicas é de 1 a 3 casos/100.000 habitantes/ano. Até recentemente era considerada uma enfermidade restrita a áreas rurais e suburbanas ${ }^{8,11-13}$. Contudo, a atividade humana vem impactando o ecossistema em que o gênero Paracoccidioides é encontrado, modificando o grau de exposição de diferentes populações humanas a esses agentes ${ }^{14}$. Outro aspecto que vem se destacando se refere aos efeitos do envelhecimento da população e o êxodo rural sobre a epidemiologia dessa doença, classicamente associada a homens com mais de 30 anos de idade e trabalhadores rurais ${ }^{4,8,15,16}$.

A paracoccidioidomicose se mostra extremamente plástica e diversificada em suas manifestações clínicas, destacando-se aquelas lesões que ocorrem no aparelho estomatognático e adjacências ${ }^{17}$ e são de responsabilidade do cirurgiãodentista no tocante ao diagnóstico e monitoramento do tratamento, uma vez que podem ser as primeiras manifestações da doença, quando não as únicas evidências de sua presença ${ }^{12}$.

Assim, considerando o exposto, o presente estudo objetivou reavaliar o conhecimento disponível sobre a etiologia, epidemiologia e manifestações clínicas da paracoccidioidomicose, reforçando o papel do cirurgião-dentista no diagnóstico das lesões bucais.

\section{MATERIAL E MÉTODO}

Esse estudo consistiu de revisão bibliográfica com análise qualitativa dos artigos obtidos. A revisão foi realizada a partir das bases de dados indexadas SciELO (Scientific Electronic Library Online) e PubMed (National Library of Medicine), optando-se por avaliar a literatura entre 1970 e 2016. Foram utilizados termos associados a "paracoccidioidomicose, etiologia, mucosa bucal, manifestações bucais, epidemiologia" e suas versões em inglês, como descritores para o levantamento. Foram desconsiderados os artigos em duplicidade e duplicidade, obteve-se um total de 1.512 artigos. Artigos adicionais em espanhol foram selecionados se completavam a avaliação em curso. Destes, foram selecionados artigos que abordavam os mecanismos envolvidos na patogênese dessa condição, a ecologia fúngica, bem como as manifestações bucais e sistêmicas da doença, selecionando-se 41 artigos que abordavam de forma abrangente essas posições.

\section{ETIOLOGIA}

A paracoccidioidomicose é uma micose sistêmica causada por fungos dimórficos do gênero Paracoccidioides, capazes de manter ciclos saprofíticos no solo, onde formam micélios típicos dos bolores, e que produzem ciclos parasitários em hospedeiros animais, como o homem, onde leveduras com gemulação múltipla se desenvolvem, sendo essa característica do fungo de grande valia para o diagnóstico microscópico da infecção ${ }^{1,4}$.

Recentemente, observou-se que a espécie $P$. brasiliensis, até então considerada a única responsável por essa micose, apresentava significativa heterogeneidade genética e parte das linhagens estudadas foi reagrupada e passou a constituir a espécie $P$. lutzii, mais frequente na região amazônica e no centro-oeste brasileiro e cuja detecção sorológica pode apresentar dificuldades adicionais ${ }^{3,4}$, uma vez que a maioria dos testes sorológicos objetiva a detecção da proteína gp43 de $P$. brasiliensis ou de anticorpos capazes de reagir com a mesma, mas a proteína equivalente de $P$. lutzii apresenta apenas $81,4 \%$ de homologia em relação ao antígeno gp43 de P. brasiliensis, o que cria condições para resultados falso-negativos e inconclusivos. Além desse aspecto, existem diferenças na expressão dos principais antígenos entre as duas espécies, afetando a resposta imunológica aos mesmos e os testes diagnósticos que objetivam a detecção da resposta de defesa contra o fungo 3,4 .

Do ponto de vista taxonômico, essas espécies fazem parte do gênero Paracoccidioides, família Ajellomycetaceae, juntamente com os fungos dos 
gêneros Blastomyces e Histoplasma, ordem Onygenales e divisão Ascomycota ${ }^{4,5}$. São fungos cujo dimorfismo está associado à temperatura, desenvolvendo-se a $25^{\circ} \mathrm{C}$ como fungo filamentoso e $37^{\circ} \mathrm{C}$ como levedura, o que pode modular a capacidade de agressão e invasão aos tecidos do hospedeiro, além da evasão da resposta imune ${ }^{3,5}$.

A caracterização do habitat desse agente no estado saprofítico é complexa, pela latência prolongada do mesmo no ambiente externo ${ }^{5}$ e em função da grande heterogeneidade de solos e climas na América Latina, bem como o efeito do impacto que a agricultura comercial e pecuária podem desempenhar na ecologia dos solos, visto que fungicidas, pesticidas e herbicidas podem dificultar o isolamento desses fungos ${ }^{18}$.

Paracoccidioides sp. apresenta predileção por solos argilosos ou arenosos ricos em matéria orgânica e ácido úrico, entre outras fontes de nitrogênio, em ambientes úmidos, clima tropical e subtropical, formando artroconídios infectantes inaláveis ${ }^{1,5}$. A relação entre o gênero Paracoccidioides e seus hospedeiros, em particular o homem, sugere que a virulência desse gênero vem apresentando uma lenta evolução, nos últimos 20-30 milhões de anos, incluindo-se aí o dimorfismo, que pode ser uma estratégia para permitir a infecção de seres endotérmicos, como os mamíferos ${ }^{5}$.

A virulência desse agente está associada à síntese de $\alpha$-(1-3)-glucano, que protege a célula fúngica da ação de enzimas lisossomais, em neutrófilos e macrófagos ${ }^{1}$, bem como a presença de diversas adesinas e produção de proteases, lipases e fosfolipases, capazes de prover os mecanismos para adesão e invasão tecidual ${ }^{5,19}$. Dentre essas enzimas destaca-se a gp43, uma proteína imunogênica capaz de degradar colágeno, caseína e elastina, além de se ligar à laminina. A presença dessa proteína ou de anticorpos voltados contra ela é estratégia mais frequente para diagnóstico sorológico dessa doença ${ }^{3}$. A mudança de fase "fungo filamentoso-levedura" também colabora na expressão dos genes de virulência e na evasão da resposta do hospedeiro ${ }^{5}$.

A fisiologia fúngica também interfere com a expressão da doença, onde a maior ocorrência de paracoccidioidomicose em homens e trabalhadores rurais possivelmente reflete a maior exposição desses aos propágulos do fungo ${ }^{8}$, bem como o efeito que os hormônios femininos, em particular $\beta$-estradiol, têm na fisiologia fúngica, inibindo a transição da fase micelial para o estado leveduriforme, nos pulmões, prevenindo o desenvolvimento da infecção ${ }^{19,20,21}$. A despeito do conhecimento disponível sobre esse gênero microbiano, o nicho ecológico por ele ocupado no solo, nas diferentes comunidades microbianas, ainda necessita de elucidação ${ }^{4}$.

\section{APRESENTAÇÃO CLÍNICO-RADIOGRÁFICA E PATOGÊNESE}

Tão diversas quanto às manifestações clínicas e radiográficas dessa doença foram as tentativas de classifica-las. A partir da germinação dos conídios inalados e a formação das células leveduriformes, a doença evolui para cura ou para quadros agudos/subagudos ou crônicos. Os quadros agudos/subagudos são mais comuns em jovens, enquanto homens adultos predominam entre aqueles com manifestações crônicas, que podem deixar sequelas mucocutâneas, ósseas, neurológicas e viscerais.

Do início do século $\mathrm{XX}$, quando a doença foi descrita por Adolf Lutz (1908), até as décadas de 1950-1960, acreditava-se que a paracoccidioidomicose se instalava pela ingestão de esporos do fungo e/ou sua introdução nos tecidos cutâneos e subcutâneos, uma vez que as lesões na mucosa bucal, laringe e faringe eram muito mais comuns do que as lesões pulmonares $^{22}$. A partir das décadas de 1960 e 1970, verificou-se que a infecção se inicia pela inalação dos esporos fúngicos, o que pode ocorrer precocemente, até os $20 \operatorname{anos}^{23}$, produzindo uma lesão inicial nos pulmões ${ }^{24-26}$, que geralmente involui ou se converte em um foco latente ${ }^{27}$. A transmissão de Paracoccidioides sp. entre humanos, por contato, não foi observada ${ }^{19}$.

Dos 10 milhões de infectados na América Latina, apenas $2 \%$ desenvolvem a doença propriamente dita ${ }^{1,19}$, enquanto, nos demais, o quadro caminha para uma infecção subclínica, para a regressão e cura, em função da maior reatividade da resposta imune celular dos portadores, ou para cronificação $^{1,17}$. Outros fatores, como a carga infectante do fungo, etilismo, tabagismo e saúde sistêmica do paciente podem afetar a progressão ou resolução da infecção ${ }^{1,23}$.

Do ponto de vista microscópico, a formação de granulomas é uma das características da resposta do hospedeiro a Paracoccidioides sp., independentemente do sítio anatômico ${ }^{1,2}$. Ao longo de todo o processo de progressão, arranjos de células gigantes multinucleadas e epitelioides se formam ao redor das leveduras com gemulação múltipla, principalmente nos pulmões, podendo levar à necrose da região central do granuloma e formação de supuração. Essa resposta passa a obedecer a determinantes típicos da resposta imune celular ${ }^{2}$. Esse complexo primário pulmonar poderá manter-se latente por muitos anos e evoluir para a remissão da infecção ou progredir dando origem a formas generalizadas e viscerais da doença ${ }^{2}$.

Os focos latentes cujo conteúdo séptico foi eliminado pelo hospedeiro sofrem remissão completa e não darão origem a relapses, mas os focos latentes 
em que fungos se mantiveram viáveis poderão dar origem às infecções crônicas, após mais de 30 anos do estabelecimento do complexo primário ${ }^{19,28}$, mantendo intensa reação cutânea a antígenos fúngicos, como a paracoccidioidina $^{2-4}$.

O complexo primário quando evolui produz infecção com manifestações clínicas inespecíficas ou mesmo sem sintomatologia. Podem ser observados casos de hemoptise, dispneia, expectoração, dor torácica, perda de peso e anorexia ${ }^{19}$, enquanto os achados radiográficos são compatíveis com infecções respiratórias de padrões variados. A partir desse foco inicial, podem ser detectadas manifestações cutaneomucosas em $10 \%$ dos indivíduos, com pápulas, vegetações e úlceras hemorrágicas ${ }^{19}$.

Como citado acima, a paracoccidioidomicose apresenta três evoluções possíveis após a infecção inicial: a remissão completa e cura, a condição aguda/subaguda ou juvenil, bem como a forma crônica ou do adulto, sendo que essa última ainda pode seguir um curso mais difuso, multifocal, com múltiplas manifestações pulmonares, ou mais restrito, com envolvimento mucocutâneo ou unifocal ${ }^{22,26}$.

A forma aguda/subaguda ou juvenil caracterizase por raramente apresentar lesões pulmonares detectáveis e envolvimento mucocutâneo frequente ${ }^{4,19}$, compreendendo de 5,0\% a 35,5\% dos $\operatorname{casos}^{29,30}$. Esses pacientes apresentam severo envolvimento do sistema mononuclear fagocitário e adrenais, além de comprometimento visceral generalizado, produzindo hepatomegalia e esplenomegalia ${ }^{13,30}$, ascite, dilatação do mediastino, lesões ósseas, articulares e icterícia ${ }^{19}$.

Essa apresentação da doença afeta jovens de ambos os sexos na mesma proporção ${ }^{17,31}$, uma vez que os primeiros estágios da infecção se desenvolvem antes do impacto do $\beta$-estradiol sobre a biologia fúngica, o que permite à infecção progredir rapidamente com disseminação linfática e/ou hematogênica em direção à pele, baço, fígado, adrenais, linfonodos e medula óssea, com raro comprometimento clínico e radiográfico de mucosas e pulmonar (as leveduras ainda podem ser detectadas por métodos moleculares nas secreções pulmonares), constituindo a forma mais grave e de prognóstico mais reservado $^{12}$, com letalidade de $11 \%$, segundo Marques $^{32}$.

A forma crônica, mais frequente em adultos (65\% a 90\% dos casos totais), é de progressão lenta, afetando principalmente indivíduos do sexo masculino e com vínculos com atividade rural, podendo ser localizada ou disseminada, com manifestações geralmente pulmonares, cutâneas e mucosas ${ }^{8,19}$.

Presentes em $70 \%$ dos pacientes adultos com paracoccidioidomicose, as lesões envolvendo laringe, faringe, mucosa bucal e região peribucal são extremamente importantes para o diagnóstico dessa enfermidade $^{17}$ e podem ser as primeiras ou únicas manifestações da doença ${ }^{12}$, o que reforça o papel do cirurgião-dentista no diagnóstico dessa enfermidade e no acompanhamento do tratamento. Tais lesões acometem principalmente os lábios, língua, gengiva e rebordo alveolar, mucosa sublingual e palato ${ }^{7,11,19}$, podendo passar sem a correta avaliação sem o exame clínico intrabucal.

$\mathrm{Na}$ mucosa bucal, as lesões geralmente são múltiplas, pápulo-erosivas, ulceradas, hipertróficas, de evolução lenta, com aspecto granulomatoso e hiperêmico, semelhantes à superfície da amora, conhecidas como "estomatite moriforme"15,33. Sialorreia, ardor, prurido e tumefação difusa podem estar presentes, além do envolvimento dos tecidos periodontais e periapicais ${ }^{7}$. Com a evolução dessa enfermidade, a laringe, faringe e tonsilas podem ser acometidas, provocando disfagia e disfonia, pelo comprometimento das cordas vocais ${ }^{19}$.

Nesses pacientes com paracoccidioidomicose residual, as sequelas mais frequentes incluem a doença pulmonar obstrutiva, disfonia, alterações nas adrenais, laringe, sistema digestório, sistema nervoso central e tegumento $^{1,2,17}$, quase sempre como consequência do processo inflamatório crônico e desenvolvimento de granulomas $^{2,19}$.

Nos pulmões, as características radiográficas evidenciam envolvimento intersticial, nodular, condensações, cavitação ${ }^{19}$, por vezes bilateral e com evidências de fibrose tecidual, devendo-se considerar também a possibilidade diagnóstica de tuberculose ${ }^{19}$, a qual também pode ser encontrada nos mesmos pacientes (de $5,5 \%$ a $19 \%$ dos casos), concomitantemente ${ }^{14,17}$.

Com o aperfeiçoamento do diagnóstico laboratorial, bem como a eficácia do tratamento medicamentoso da paracoccidioidomicose, a ocorrência de quadros mais graves vem diminuindo progressivamente, mas pacientes com severa perda de peso, insuficiência respiratória, envolvimento de suprarrenais e do sistema nervoso central ainda podem ser observados ${ }^{17}$.

Dependendo da localização das lesões e sua apresentação clínico-radiográfica, no diagnóstico diferencial deve-se incluir também a histiocitose, tuberculose, hanseníase, cromoblastomicose, leishmaniose mucocutânea, carcinoma espinocelular, queimaduras químicas, actinomicose, sarcoidose, granulomatose de Wegener e doenças autoimunes $^{17,19,34}$.

\section{EPIDEMIOLOGIA DA}

\section{PARACOCCIDIOIDOMICOSE}

Por não ser uma doença de notificação compulsória, os dados epidemiológicos relativos à paracoccidioidomicose são restritos ${ }^{7,15,35}$. Apesar de 
endêmica na América Latina, do sul do México até a Patagônia, os estudos epidemiológicos sobre a doença são limitados ${ }^{10,15,27,29,35}$.

A maior ocorrência dessa micose profunda no Brasil possivelmente reflete múltiplos fatores, como as condições propícias de solo e clima, existência de uma agricultura comercial intensiva, e a grande população até recentemente em contato com atividadesagropecuárias, fazendo do território brasileiro uma área superendêmica para esse fungo ${ }^{29}$.

A paracoccidioidomicose é a micose sistêmica de maior relevância no território nacional ${ }^{26} \mathrm{e}$ na América Latina ${ }^{2}$, responsável por $51,2 \%$ dos óbitos ligados às micoses profundas no Brasil ${ }^{3}$, além de se apresentar frequentemente em outros países da região, como Colômbia e Venezuela ${ }^{4,27}$. Por outro lado, devese destacar a baixa ocorrência dessa micose na América Central e sua ausência no Chile, Antilhas e Guianas $^{19,35}$.

No Brasil, uma ocorrência mais elevada dessa infecção vem sendo descrita nos estados de São Paulo, Rio Grande do Sul, Paraná, Rio de Janeiro, Goiás, Rondônia, Espírito Santo, Minas Gerais, Mato Grosso do Sul, Mato Grosso, Amazonas e Maranhão ${ }^{2,4,8-10,19}$, onde até $50 \%$ da população de áreas endêmicas foram expostos a Paracoccidioides sp. e apresentam reação à paracoccidioidina $^{2,23}$. Sua ocorrência é da ordem de 1 a 3 casos/100.000 habitantes/ano, com mortalidade da ordem de 0,14 a 4,49 casos/100.000 habitantes/ano ${ }^{1,2,16,29}$, com grande heterogeneidade dentro do território nacional.

As sequelas se desenvolvem em aproximadamente um terço dos pacientes tratados e o óbito sobrevém para 2 a $23 \%$ dos mesmos, com média de $7,6 \%{ }^{2,13}$, constituindo a quinta causa mais relevante de óbitos em pacientes portadores do vírus $\mathrm{HIV}^{36}$, onde sua letalidade pode se elevar a mais de $30 \%^{2}$, o que reforça a necessidade de cuidados adicionais no diagnóstico e tratamento dessa micose nesses pacientes $^{37}$. Como agravante, na região sudeste do Brasil, 1,4\% dos indivíduos HIV-positivos são portadores de paracoccidioidomicose e, entre esses, os trabalhadores rurais são $\operatorname{raros}^{27}$. Nos pacientes HIVpositivos com níveis de linfócitos $\mathrm{T}_{4}$ inferiores a 200 $\mathrm{cel} / \mathrm{mm}^{3}$ a forma aguda/subaguda é muito mais frequente $^{37}$.

Em algumas regiões do Brasil, como nas bordas da Amazônia Legal, a infecção por Paracoccidioides sp. (incluindo $P$. brasiliensis e $P$. lutzii) é bastante frequente, atingindo 10,8 casos/100.000 habitantes/ano no Maranhão ${ }^{9}$, 9,4 casos/100.000 habitantes/ano em Rondônia, sendo que os municípios de Pimenteiras do Oeste e Espigão do Oeste, frentes agrícolas importantes, apresentaram uma casuística bastante intensa, da ordem de 39,1 casos/100.000 habitantes/ano e 37,4 casos/100.000 habitantes/ano, respectivamente ${ }^{8,10}$.

Desde o final do século XX tem-se observado um significativo aumento no diagnóstico de casos de paracoccidioidomicose no Brasil. Essas informações epidemiológicas apontam para a importância das fronteiras agrícolas do país ${ }^{2}$, mas também para um aumento da sensibilidade e especificidade dos testes de diagnóstico sorológico e a problemática da infecção concomitante pelo vírus $\mathrm{HIV}^{8}$.

O predomínio do sexo masculino entre os pacientes com paracoccidioidomicose é bastante evidente ${ }^{4,7,11,14,27,34,35}$, sendo que a relação sexo masculino/feminino varia de 5,4:1 a 30:1, como evidenciado na literatura $8,11,14,15,34$. Este fenômeno pode estar relacionado a dois fatores principais: a maioria dos infectados é constituída por trabalhadores rurais $^{8,15,22,26,35}$, que geralmente são do sexo masculino, mas também é reconhecida proteção natural que as mulheres possuem em função do $\beta$ estradiol, que pode afetar a transição da fase micelial para a leveduriforme nesses fungos ${ }^{11} \mathrm{e}$ alterar a resposta imune celular ${ }^{11,21}$. Deve-se ressaltar ainda que a ocorrência de paracoccidioidomicose entre as mulheres vem diminuindo ${ }^{14}$.

A paracoccidioidomicose, quando se manifesta no período que antecede a puberdade, não apresenta a marcada predileção pelo sexo masculino observada na forma crônica. Isso possivelmente se dá pelo fato de que as meninas ainda não produzem níveis significativos de $\beta$-estradiol capazes de inibir a transição do fungo para a fase leveduriforme ${ }^{19}$. Nas demais faixas etárias, em função das modificações do sistema endócrino, as mulheres seriam menos atingidas, representando menos de $10 \%$ dos pacientes com a forma crônica e progressiva da doença ${ }^{8,19}$. Após a menopausa, as mulheres se mostram mais suscetíveis à doença, com níveis próximos aos observados com homens, daí a importância da investigação do nível de estrógeno quando da suspeita de paracoccidioidomicose em mulheres ${ }^{12}$.

Aparentemente todos os grupos étnico-raciais apresentam a mesma susceptibilidade à infecção ${ }^{35}$, que ocorre com maior frequência entre a terceira e quinta décadas de vida, com alguns casos sexta década ${ }^{4,8,15,16,19,23,26}$, embora Blotta et al. ${ }^{34}$, analisando 584 casos na região sudeste, encontraram maior incidência da doença entre a segunda e a quarta décadas de vida.

A paracoccidioidomicose é uma doença associada principalmente a indivíduos procedentes da área rural ou a ela ligados mesmo que indiretamente, como jardinagem e transporte de produtos vegetais $^{8,23,29}$. Entretanto, observa-se também um número elevado de ocorrências entre pessoas com 
outras atividades profissionais, com aumento progressivo da doença em regiões suburbanas e urbanas $^{6,11-13}$.

Essa relação entre atividade rural e ocupação do solo tem impactos que duram anos na epidemiologia da paracoccidioidomicose. No sul do Brasil, Argentina e Paraguai, os quadros agudos/subagudos da paracoccidioidomicose são raros e quase todos os informes epidemiológicos evidenciam a universal predominância da forma crônica, em homens adultos $^{38-40}$, sugerindo que aspectos ecológicos, climáticos e/ou geológicos podem interferir com a distribuição clínica da doença ${ }^{14}$.

É possível que o desmatamento, aumentando o conteúdo de Paracoccidioides sp. no ambiente e exacerbando o contato entre populações susceptíveis com o agente, pode resultar em um aumento da ocorrências das formas mais agudas e agressivas da doença nas áreas de colonização recente, o que não ocorre na região Sul do Brasil e na Bacia Platina como um todo, onde a ocupação humana é intensa, estável e mais antiga, e onde desmatamento de grandes áreas constitui problema do passado, ao passo que esse fenômeno pode ser de grande impacto na região amazônica, em particular no arco do desmatamento, que compreende os estados de Rondônia, Pará, Acre, Mato Grosso e Maranhão ${ }^{14}$, que constituem as frentes pioneiras de ocupação no Sul e Leste da Amazônia Legal $^{23,35}$.

Outro aspecto que pode estar contribuindo para a diminuição dos casos agudos entre os jovens é a redução do trabalho infantil e o envelhecimento da população rural, reduzindo significativamente a exposição da população infantil e juvenil aos conídios inaláveis de $P$. brasiliensis e $P$. lutzii ${ }^{14}$.

Além do solo, Paracoccidioides sp. tem sido detectado em diferentes espécies animais, como tatus $^{1,41}$, morcegos ${ }^{1,41}$, fezes de pinguins $^{5}$, em guaxinins, furões, cobaias, porcos-espinho ${ }^{41}$ e cães suburbanos, geralmente nos pulmões e vísceras, onde produzem alterações histológicas típicas da paracoccidioidomicose $\mathrm{e}^{1,5,41}$ e podem participar do ciclo da doença em humanos.

\section{CONCLUSÃO}

Os dados apresentados reforçam o papel do cirurgião-dentista no diagnóstico da paracoccidioidomicose, em particular a sua forma crônica, que concentra nas mucosas grande parte de suas manifestações clínicas e produz envolvimento tecidual capaz de desfigurar o paciente, comprometendo sua qualidade de vida e, acima de tudo, evoluir para o óbito ou sequelas graves. Além desse aspecto, essa micose tem mostrado uma significativa modificação em seus padrões epidemiológicos, refletindo a profunda transformação do espaço econômico e das populações que habitam as regiões endêmicas, em particular nas regiões Norte e Centro-Oeste do Brasil, que constituem as mais promissoras fronteiras agrícolas do país, sugerindo a necessidade de reavaliações periódicas dos dados disponíveis sobre ocorrência e manifestações da doença.

\section{REFERÊNCIAS}

1. Moreira APV. Paracoccidioidomicose: histórico, etiologia, epidemiologia, patogênese, formas clínicas, diagnóstico laboratorial e antígenos. BEPA Bol epidemiol Paul. 2008;5(51):10-24.

2. Góes AM, Silva LSS, Araújo SA, Cruz SG, Siqueira WC, Pedroso ERP. Paracoccidioidomycosis disease (Lutz-SplendoreAlmeida): etiology, epidemiology, and pathogenesis. Rev méd Minas Gerais. 2014;24(1):58-63.

3. Taborda CP, Urán ME, Nosanchuk JD, Travassos LR. Paracoccidioidomycosis: challenges in the development of a vaccine against an endemic mycosis in the Americas Rev Inst Med Trop Sao Paulo 2015;57(Supl. 19):21-4.

4. Silva JF, Oliveira HC, Marcos CM, Assato PA, Fusco-Almeida AM, Mendes-Giannini MJS. Advances and challenges in paracoccidioidomycosis serology caused by Paracoccidioides species complex: an update. Diagc Microbiol Infect Dis. 2016;84(1):87-94.

5. Bagagli E, Theodoro RC, Bosco SMG, McEwen JG. Paracoccidioides brasiliensis: phylogenetic and ecological aspects. Mycopathologia. 2008;165(4-5):197-207.

6. Vieira EM, Borsatto-Galera B. Manifestações clínicas bucais da paracoccidiodomicose. Rev Patol Trop. 2006;35(1):23-30.

7. Araújo MS, Sousa SCM. Análise epidemiológica de pacientes acometidos com paracoccidiodomicose em região endêmica do Estado de Minas Gerais. Rev Pos-Grad. 2000;7(1):22-6.

8. Souza SP, Jorge VM, Xavier MO. Paracoccidioidomycosis in southern Rio Grande do Sul: a retrospective study of histopathologically diagnosed cases. Braz J Microbiol. 2014;45(1):243-7.

9. Matos WB, Santos GMC, Silva VEB, Gonçalves EGR, Silva AR. Paracoccidioidomycosis in the state of Maranhão, Brazil: geographical and clinical aspects. Rev Soc Bras Med Trop. 2012;45(3):385-9.

10. Vieira GD, Alves TC, Lima SMD, Camargo LMA, Sousa CM. Paracoccidioidomycosis in a 
western Brazilian amazon state: clinicalepidemiologic profile and spatial distribution of the disease. Rev Soc Bras Med Trop. 2014;47(1):63-8.

11. Hassessian A, Ishikawa EN, Alencar FI, Marcucci, G. Estudo da prevalência de lesões bucais em pacientes portadores de paracoccidioidomicose na região de Campo Grande - Mato Grosso do Sul. Rev Pos-Grad. 2000;7(3):214-8.

12. Almeida OP, Jorge Jr J, Scully C. Paracoccidioidomycosis of the mouth: an emerging deep mycosis. Crit Rev Oral Biol Med. 2003;14(5):268-74.

13. Paniago AMM, Aguiar JIA, Aguiar ES, Cunha RV, Pereira GROL, Londero AT, et al. Paracoccidioidomicose: estudo clínico e epidemiológico de 422 casos observados no Estado de Mato Grosso do Sul. Rev Soc Bras Med Trop. 2003;36(4):455-9.

14. Fabris LR, Andrade UV, Santos AF, Marques APC, Oliveira SMVL, Mendes RP, et al. Decreasing prevalence of the acute/subacute clinical form of paracoccidioidomycosis in Mato Grosso do Sul state, Brazil. Rev Inst Med Trop Sao Paulo 2014;56(2):121-5.

15. França DCC, Monti LM, Castro AL, Soubhia AMP, Aguiar SMHCA. Análise retrospectiva de 61 casos de paracoccidioidomicose diagnosticados na Faculdade de Odontologia de Araçatuba/ UNESP. Rev Odontol Araçatuba 2011(1);32:9-14.

16. Carvalhosa AA, Borges FT, França DCC, Queiroz RR, Moimaz ASS, Garbin CAS. Paracoccidioidomycosis prevalence in a public laboratory of the Brazilian unified health system. J Oral Diag. 2012;1(1):31-5.

17. Wanke B, Aidê MA. Paracoccidioidomicose. J Bras Pneumol. 2009;35(12):1245-9.

18. Ono MA, Itano EN, Mizuno LT, Mizuno EHF, Camargo ZP. Inhibition of Paracoccidioides brasiliensis by pesticides: is this a partial explanation for the difficulty in isolating this fungus from the soil? Med Mycol. 2002;40(5):493-9.

19. Ramos-E-Silva M, Saraiva L do E. Paracoccidioidomycosis. Dermatol Clin. 2008;26(2):257-9.

20. Felipe MSS, Torres FAG, Maranhão AQ, SilvaPereira I, Poças-Fonseca MJ, Campos EG, et al. Functional genome of the human pathogenic fungus Paracoccidioides brasiliensis. FEMS Immunol Med Microbiol 2005;45(3):369-81.

21. Shankar J, Restrepo A, Clemons KV, Stevens DA. Hormones and the resistance of women to paracoccidioidomycosis. Clin Microbiol Rev. 2011;24(2):296-313.
22. Lemle A, Vieira LOBD, Milward GAF, Miranda JL. Lung functions studies in pulmonar South American blastomycosis. Amer J Med. 1970;48(4):434-42.

23. Shikanai-Yasuda MA, Telles Filho FQ, Mendes RP, Colombo Al, Moretti ML, Consenso em paracoccidiodomycosis. Rev Soc Bras Med Trop. 2006;39(3):297-310.

24. Negroni P, Negroni R. Nuestra experiencia de la blastomicosis sudamericana en la Argentina. Mycopathologia 1965;26(2):264-72.

25. Restrepo A, Robledo M, Gutierrez F, Sanclemente M, Castaneda E, Calle G. Paracoccidioidomycosis (South American blastomycosis). A study of 39 cases observed in Medellin, Colombia. Amer J Trop Med Hyg. 1970;19(1):68-76.

26. Londero AT, Ramos CD. Paracoccidioidomycosis. A clinical and mycologic study of forty-one cases observed in Santa Maria, RS, Brazil. Amer J Med. 1972;52(6):771-5.

27. Marques SA. Paracoccidioidomycosis. Clin Dermatol. 2012;30(6):610-5.

28. Sylvestre TF, Silva LRF, Cavalcante RS, Moris DV, Venturini J, Vicentini AP, et al. Prevalence and serological diagnosis of relapse in paracoccidioidomycosis patients. PLoS Negl Trop Dis. 2014;8:e2834.

29. Bellissimo-Rodrigues F, Machado AA, Martinez R. Paracoccidioidomycosis epidemiological features of a 1,000-cases series from a hyperendemic area on the Southeast of Brazil. Amer J Trop Med Hyg. 2011;85(3):546-50.

30. Nogueira MG, Andrade GM, Tonelli E. Clinical evolution of paracoccidioidomycosis in 38 children and teenagers. Mycopathologia 2006;161(2):73-81.

31. Coutinho ZF; Silva D, Lazéra M, Petri V, Oliveira RM, Sabroza PC, et al. Paracoccidioidomycosis mortality in Brazil (1980-1995). Cad. Saúde Pública 2002;18(5):1441-54.

32. Marques SA. Paracoccidioidomicose. An Bras Dermatol. 1998;73:455-69.

33. Weismann R, Rosa ELS, Lima HVO, Veloso, MGP, Miziara, HL. Paracoccidioidomicose: relato de caso. Rev Odonto Ciência. 1995;20:203-11.

34. Blotta, MH, Mamoni RL, Oliveira SJ, Nouér AS, Papaiordanou PM, Gouveia A, et al. Endemic regions of paracoccidioidimycosis in Brazil: a clinical and epidemiologic study of 584 cases in the southeast region. Am J Trop Med Hyg. 1999;61(3):390-4.

35. Martinez R. Epidemiology of paracoccidioidomycosis. Rev Inst Med Trop Sao Paulo 2015;57(supl. 19):11-20.

36. Prado M, Silva MB, Laurenti R, Travassos LR, 
Taborda CP. Mortality due to systemic mycoses as a primary cause of death or in association with AIDS in Brazil: a review from 1996 to 2006. Mem Inst Oswaldo Cruz. 2009;104(3):513-21.

37. Safe IP, do Valle FF, Maia DCC, Agonio B, Monte RL, Araújo JR, et al. Extra-pulmonary manifestations of paracoccidioidomycosis associated with acquired immunodeficiency syndrome: a case report. An Bras Dermatol. 2014;89(1):150-3.

38. Rolon PA. Paracoccidioidomicosis. Epidemiologia en la República del Paraguay, centro Sud América. Mycopathologia 1976;59(2):67-80.

39. Gimenez MM, Storni LP, Gimenez MF. Paracoccidioidomicosis. Nuestra experiencia. Rev Argent Dermatol. 1984;65:273-9.

40. Colares SM, Marcantônio S, Zambonato S, Severo LC. Acute/subacute disseminated paracoccidioidomycosis. First case in Rio Grande do Sul State, Brazil. Rev Soc Bras Med Trop. 1998;31(6):563-7.

41. Richini-Pereira VB, Bosco SMG, Griese J, Theodoro RC, Macoris SAG, Silva RJ, et al. Molecular detection of Paracoccidioides brasiliensis in road-killed wild animals. Med Mycol. 2008; 46(1):35-40

\section{CONFLITO DE INTERESSES}

Os autores declaram não haver conflitos de interesse.

\section{AUTOR PARA CORRESPONDÊNCIA}

Elerson Gaetti Jardim Júnior

egaetti@foa.unesp.br
Submetido em 31/03/2016 Aceito em 08/04/2016 